\title{
Regards croisés sur l'éducation en Chine
}

\section{Kechao Xing et Pierre-Louis Gauthier}

\section{CpenEdition}

\section{Journals}

Édition électronique

URL : http://journals.openedition.org/ries/3168

DOI : 10.4000/ries.3168

ISSN : 2261-4265

\section{Éditeur}

Centre international d'études pédagogiques

\section{Édition imprimée}

Date de publication : 1 septembre 1997

Pagination : 61-68

ISSN : 1254-4590

\section{Référence électronique}

Kechao Xing et Pierre-Louis Gauthier, «Regards croisés sur l'éducation en Chine », Revue internationale d'éducation de Sèvres [En ligne], 15 | 1997, mis en ligne le 04 juillet 2013, consulté le 20 avril 2019. URL : http://journals.openedition.org/ries/3168 ; DOI : 10.4000/ries.3168

Ce document a été généré automatiquement le 20 avril 2019

(c) Tous droits réservés 


\title{
Regards croisés sur l'éducation en Chine
}

\author{
Kechao Xing et Pierre-Louis Gauthier
}

1 Il n'est pas concevable d'examiner les structures d'un système éducatif dans quelque pays que ce soit sans tenir compte des conditions géographiques et économiques ainsi que de la tradition culturelle et historique de ce pays.

2 Les dimensions de la Chine, le volume et la diversité de sa population font que tout phénomène humain provoque dans ce pays une résonance et entraîne des conséquences d'une ampleur difficile à imaginer dans les pays occidentaux. A fortiori lorsque, dans un contexte de réformes et de changements rapides, évolutifs et irréversibles, se produisent des phénomènes éducatifs.

3 Cette évolution liée aux récents bouleversements économiques s'accompagne de décisions gouvernementales dans le domaine éducatif, du préscolaire jusqu'à l'université. Ainsi, le budget de l'éducation devrait atteindre, en principe, $4 \%$ du PIB en l'an 2000 contre $2,7 \%$ actuellement. On mesure ainsi l'ampleur de l'évolution du système éducatif dans ce contexte. Mais on peut s'interroger également sur la capacité des décideurs et des acteurs de cette évolution à en maîtriser tous les développements et notamment les inégalités qui la caractérisent. 


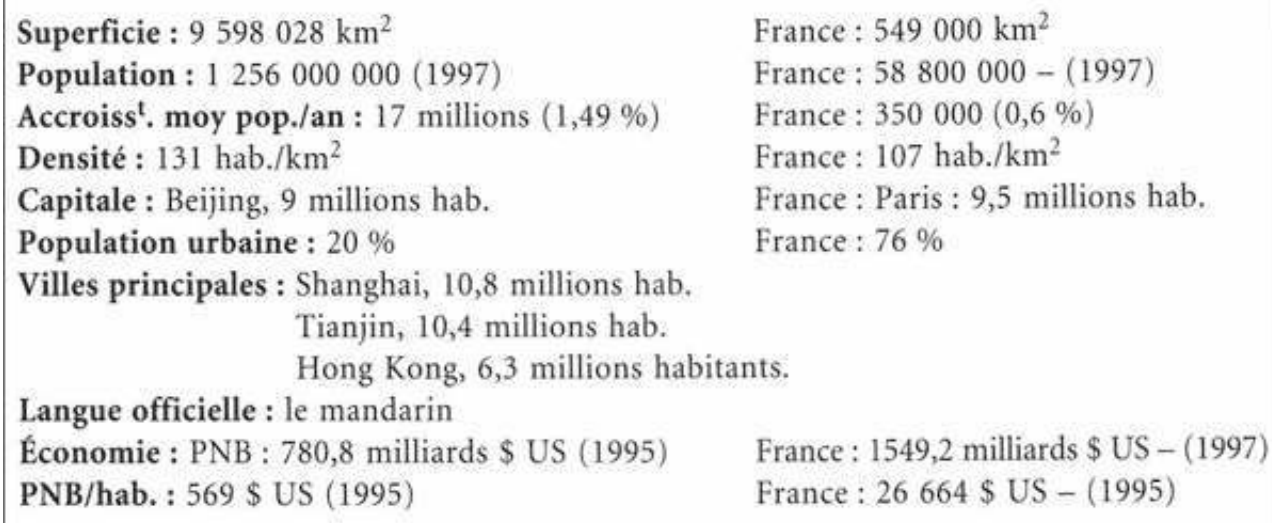

Cet article n'a pas pour but de décrire de manière exhaustive le système éducatif chinois, mais plutôt de fournir au lecteur un aperçu de la problématique actuelle et des principaux dossiers qui s'imposent aux enseignants comme à l'ensemble de l'administration et des dirigeants politiques dans l'inéluctable mutation de la Chine actuelle.

\section{Entre centralisation et autonomie}

\section{Tradition et modernité}

La tradition unitaire de la Chine plonge ses racines dans la fondation même du Premier Empire (II ${ }^{e}$ siècle avant notre ère). La notion d'État qui émerge dès cette lointaine époque va de pair avec le système de recrutement et de formation des fonctionnaires impériaux. L'administration se fonde donc sur l'éducation. Mais ce système se fige très tôt, devenant itératif en dépit d'une lente évolution jusqu'à la fin de la dernière dynastie des Qings, au début du XX $X^{\mathrm{e}}$ siècle.

6 La formation reste étroitement liée au recrutement des fonctionnaires qui s'opère à travers une stricte sélection indéfiniment répétée aux différents niveaux de l'administration, cantonale, provinciale ou nationale. La progression des contenus de la formation, identique à travers tout le continent chinois, consiste en un nombre important de textes formels, de listes, dont la plupart doivent être mémorisés.

7 La consécration finale pour le futur mandarin reste l'épreuve nationale de la dissertation littéraire ou baguwen, rigidifiée en un exercice de style en huit parties qui se déroule selon un rite immuable, dans un édifice de la capitale réservé à cet usage.

8 De fait, en dépit des mouvements révolutionnaires et du charisme de leurs dirigeants (Sun Yat Sen en 1911 et Mao Zedong en 1949), le principe général d'unification du pays, y compris en matière d'éducation, est resté inchangé jusqu'à la fin de la révolution culturelle (1976). Les premiers changements importants interviennent à partir de 1978 corrélativement à la politique de réforme et d'ouverture. À partir de cette date, la priorité passe du terrain idéologique et politique à l'édification d'une économie nouvelle dite "économie socialiste de marché » (décisions du Comité central du parti communiste 
chinois de décembre 1978). Le slogan " expert puis rouge ", succédant au "rouge puis expert » de la révolution culturelle, résume cette évolution.

Comparativement à cette mise en mouvement de l'économie et de la société chinoise, le caractère figé de l'éducation apparaît d'autant plus évident. Dès lors, les réformes du système éducatif s'imposent dans la perspective des "modernisations " annoncées dès 1980 par Deng Xiaoping.

10 Au cours des années suivantes, une série de mesures aboutissent progressivement à une déconcentration des niveaux de décision. Mais, parallèlement, les types d'organisation de l'enseignement se diversifient à travers le pays. Actuellement, on peut rencontrer des structures d'enseignement très différentes suivant les régions. Par exemple, des écoles primaires de 4, 5 ou 6 années, des écoles de base "primaire +2 années » (au lieu de " primaire +3 années »), des écoles normales d'une scolarité de 3,4 ou 5 années, suivant les districts et leurs possibilités, etc.

11 Pour l'enseignement supérieur, une centaine d'universités ou instituts d'enseignement supérieur provinciaux ont été fondés en quelques années. On envisage de leur déléguer la collation des grades au niveau des provinces à partir de 1997. Si les programmes restent nationaux, dépendants directement de la commission d'État pour l'éducation (l'équivalent du ministère), les manuels par contre peuvent être rédigés et publiés aux niveaux provincial et municipal.

12 Ce processus de décentralisation (fenquan) et déconcentration (fang-quan) rencontre des obstacles dans la mentalité même des administrateurs aussi bien que dans celle des praticiens. D'une part, on a plutôt l'habitude de donner des ordres souvent très concrets et précis - et /ou d'obéir passivement; d'autre part, on pourra également réclamer une liberté totale. On donne donc une expression significative à ces deux extrêmes en disant: " entre rigidité ou désordre ». C'est ainsi que de nombreux instituts provinciaux ont pris ces dernières années le nom d'université sans pour autant aboutir à une réelle amélioration des contenus et de la qualité de l'enseignement, créant une certaine confusion parmi les compétences. Ailleurs, aucune modification ni des structures, ni des contenus n'est sensible. Le gouvernement central, les autorités locales ainsi que les établissements auront beaucoup à faire pour réaliser une cohésion organique et optimale entre la centralisation et l'autonomie.

\section{Une, deux, trois Chines?}

\section{But global et disparités régionales}

13 Sur le fond d'unité nationale, séculaire et profondément ancrée dans l'esprit des Chinois, il a toujours existé en fait de profondes disparités régionales ${ }^{1}$. Actuellement, si au plan politique la cohésion et l'unité nationales restent fermement assurées, on peut, économiquement et culturellement, distinguer trois zones bien diversifiées sur le territoire chinois.

14 À l'est, une zone couvrant les régions côtières à forte densité de population connaît depuis longtemps un développement économique et culturel qui s'accompagne actuellement d'un accroissement rapide du PIB. Le centre de cette zone est situé autour de la ville de Shanghai, dans les provinces de Zhejiang et Jiangsu. À cette zone de développement, il convient d'adjoindre respectivement, dans le nord, Beijing, la capitale 
nationale, et sa région immédiate, et dans le sud, le Guangdong et sa capitale Canton. Les dix districts les plus riches de la Chine se trouvent dans ces régions.

À l'ouest, une zone très contrastée avec la précédente, peu peuplée, surtout habitée par la plupart des cinquante-quatre minorités ethniques (Ouighours, Tibétains, Miao, Zhuangs...) et où les conditions naturelles sont défavorables (montagnes, déserts). Cette zone n'a jamais connu de fort développement économique en dépit de ses ressources minières. Actuellement, le PIB reste faible et est au moins dix fois inférieur à celui de la zone orientale. Il s'agit principalement des provinces du Xinjiang, Tibet, Ningxia, Qinghai, Gansu, Yunnan, Guizhou.

Entre ces deux parties, une zone centrale regroupe des provinces au développement moyen. Il s'agit du Hubei, Henan, Shanxi, Hunan.

La réforme économique de 1978 a eu pour effet d'amplifier les disparités déjà anciennes entre ces zones. Dans le domaine de l'éducation ces disparités sont criantes. Ainsi, alors que les écoles de l'est s'équipent avec des ordinateurs et des laboratoires de langues, une partie de celles de l'ouest en sont encore à tenter de garantir un minimum de sécurité en matière de bâtiments et à rechercher les moyens d'un équipement élémentaire en mobilier scolaire.

18 Face à la disparité croissante, le gouvernement conduit une politique de généralisation progressive des neuf années de scolarité obligatoire qui devrait, d'ici à l'an 2010, être réalisée selon le plan suivant :

- dans les régions riches et moyennes où se regroupent $85 \%$ de la population chinoise, la scolarité obligatoire serait portée à 9 années ;

- dans les régions pauvres où se trouve $10 \%$ de la population, la scolarité obligatoire serait uniquement généralisée dans le primaire (5-6 années);

- dans les régions les plus pauvres où se trouve le reste de la population (5\%), la scolarité obligatoire atteindrait seulement 3 à 4 années.

En outre, l'État encourage les conventions entre régions favorisées et régions défavorisées ou entre districts et établissements (jumelages). Des fonds spéciaux, appelés "travaux d'espoir» (xiwanggongcheng), sont accordés dans ce but. Le système de l'orientation préalable devrait permettre aux étudiants de revenir dans leur province d'origine après leurs études².

Les mesures adaptées à un développement fortement inégal n'atteignent cependant pas facilement leur but, d'autant que les régions défavorisées connaissent une émigration importante, déjà ancienne, et en particulier une fuite des cerveaux vers l'est.

\section{Dans l'enseignement général}

\section{Le poids des examens}

21 Issu directement de la tradition mandarinale, l'examen en Chine constitue toujours le but ultime et principal des études. Après 1949, en dépit d'une élévation rapide du taux de scolarisation, les universités n'avaient pas la possibilité de recevoir beaucoup d'étudiants et on dut maintenir l'examen d'entrée à l'université. Mais, à côté de ce modèle prestigieux, on retrouve maintenant la procédure de l'examen à tous les moments de la vie scolaire. Comme jadis dans la formation des fonctionnaires impériaux, une sélection 
permanente pèse de tout son poids sur l'organisation des études et la conception de l'enseignement.

22 Ce poids de l'examen influe négativement sur l'enseignement, accordant une place primordiale à la mémorisation et à la théorisation et négligeant la formation des compétences et des aptitudes qui sont pourtant largement aussi utiles.

23 Cette tendance dévoie l'enseignement vers des techniques d'entraînement orientées essentiellement vers la réussite à l'examen. Ainsi, primauté est accordée à la forme, à l'étude du titre de l'épreuve, au repérage des points importants. La notation est poussée à plusieurs décimales par les enseignants, les disciplines nécessaires à l'examen (chinois, mathématiques, anglais) sont valorisées à l'extrême, aux dépens des disciplines «inutiles» à l'examen (biologie par exemple) qui sont négligées, voire pratiquement éliminées.

Actuellement, où la recherche d'un emploi très qualifié, dans une économie plus performante, s'ajoute au prestige social traditionnel de l'examen, celui-ci s'en trouve encore renforcé. Les enfants chinois entrent en concurrence les uns avec les autres, dès leur première année scolaire et même avant. Compte tenu qu'il existe des disparités entre les établissements d'une même province, voire d'une même ville, le choix des écoles présente une importance primordiale pour l'orientation universitaire et professionnelle future de l'élève. En effet, le lycée détermine souvent de manière irréversible cette orientation et on dit en Chine qu'il est plus difficile d'entrer dans un bon lycée qu'à l'université. On emploie alors tous les moyens pour échapper à la sectorisation.

Les conséquences de la prégnance de l'examen sur les enfants et les adolescents sont souvent négatives: à la masse de devoirs, à la négligence de l'éducation physique, artistique et morale, s'ajoute un esprit de compétition trop précoce. On constate aussi des conséquences psychosomatiques comme le développement de la myopie et certaines formes de stress. Les élèves de niveau moyen ou médiocre, comprenant très tôt au fil des nombreux examens qu'ils n'accéderont jamais à l'enseignement supérieur, rencontrent très vite le désintérêt pour les études, voire la désespérance en classe.

En outre dans la famille, l'enfant unique, issu de la politique de réduction de la natalité, se trouve investi de tous les vœux de réussite et frustrations de ses parents et grandsparents. Cet "enfant-roi » - on dit en Chine "petit empereur" (xiaohuangdi) - réagit souvent par l'insolence, la paresse, le caprice, à ces trop fortes pressions.

Cette problématique de l'examen provoque actuellement un large débat en Chine et entraîne dans beaucoup d'écoles de nombreuses expériences alternatives.

\section{Dans l'enseignement supérieur}

\section{Des choix improbables}

Créé à la fin du siècle dernier sur le modèle américain dans les grandes villes, l'enseignement supérieur chinois de type moderne n'a pas connu de grands développements avant 1949. Après la fondation de la République populaire de Chine, le gouvernement a nationalisé les universités qui étaient auparavant, pour la plupart, privées et leur a adapté le modèle soviétique: nombreux instituts, filières très spécialisées. Actuellement, compte tenu de l'évolution scientifique, technique, économique et sociale, ce modèle universitaire ne correspond plus aux besoins du pays et 
une réforme profonde est devenue nécessaire et inévitable. On peut la résumer pour l'instant en trois points.

\section{Moins d'universités et plus d'étudiants}

Dans le but d'une utilisation optimale des ressources humaines et matérielles, on vise au seul maintien du nombre des universités, voire à leur réduction (on comptait 1064 établissements d'enseignement supérieur ordinaires en 1996). Le nombre d'étudiants passerait, lui, de 5,5 millions ${ }^{3}$ à 6,5 millions de 1996 à l'an 2000. Dans cet effort d'optimisation de la structure, le développement de l'enseignement supérieur doit pourtant rester équilibré eu égard aux besoins incomparablement plus importants de l'enseignement de base. Or il apparaît que la tendance à la chasse individualiste au diplôme tende à l'emporter sur des considérations d'ordre général. Comme il y a entre les intentions gouvernementales et les mentalités de la population un décalage important, le développement récent des premiers cycles universitaires (dazhuan), quoique encore insuffisant, aboutit déjà en 1997 à une inflation de diplômes.

\section{Des filières moins nombreuses et plus larges}

L'héritage de la période soviétique, constitué de 813 filières minutieusement cloisonnées, évolue vers le réaménagement de 504 filières seulement, offrant des combinaisons moins nombreuses. Les filières liées à l'économie (gestion, droit, informatique), ainsi que les sciences appliquées et les sciences de pointe, se développent plus rapidement.

\section{Moins de centralisation et plus de coopération verticale et horizontale}

31 Le niveau de décision est ramené du national au provincial ou au municipal, l'État se réservant les macrodécisions dans les domaines de l'évaluation, de la planification, de la formation. On veille néanmoins à maintenir une coopération verticale entre ces différents niveaux. Par exemple, la commission d'État pour l'éducation passe des contrats avec une ville, une province ou un ministère pour gérer ensemble des universités qui appartenaient autrefois exclusivement à l'une ou à l'autre partie.

En termes de coopération horizontale, il y a trois orientations :

- la coopération entre des universités de haut niveau et des universités plus modestes ;

- la coopération entre des universités relevant de différents ministères (sidérurgie, pétrole, télécommunications, agronomie, électronique), possible uniquement dans les grandes villes où existe une certaine concentration universitaire ;

- l'orientation «enseignement-recherche-production" tendant à promouvoir l'économie locale et à augmenter le budget propre de l'université qui est souvent très modeste par rapport aux besoins.

\section{Entre urgence et désaffection}

\section{Un corps enseignant quantitativement et qualitativement insuffisant}

Pour assurer le développement de l'éducation nationale, il faut avoir un corps d'enseignants qui soit quantitativement et qualitativement adapté. Quantitativement, la 
Chine manque d'enseignants. Actuellement, les conditions de l'enseignement connaissent des variations considérables dans les différentes zones du pays et d'une manière générale, la pénurie d'enseignants domine. Il faut donc encore recruter dans les villages des «enseignants-paysans » (minbanjiao shi), rémunérés partie par l'État, partie par les villageois et qui sont d'un niveau parfois inquiétant ( $28 \%$ des enseignements du primaire et 5,96\% des enseignements des collèges étaient assurés par des " enseignants-paysans " fin 1995).

Parmi les enseignants à plein temps, certains n'ont pas atteint le niveau de qualification requis par l'État, à savoir :

- pour les instituteurs : enseignement obligatoire de neuf années + trois années de formation à l'école normale ( $88,9 \%$ des instituteurs ont atteint le niveau requis);

- pour les professeurs de collège : diplôme du $1^{\text {er }}$ cycle universitaire $(69,1 \%$ ont atteint le niveau requis);

- pour les professeurs de lycée : diplôme de $2^{\mathrm{e}}$ cycle universitaire ( $55,2 \%$ ont atteint le niveau requis).

Pour attirer davantage de jeunes candidats à l'enseignement, l'État a augmenté les salaires et amélioré les conditions de travail et de vie. On a pratiqué une formation «pré orientée " permettant au candidat à l'enseignement de connaître avant son engagement et sa période de formation son futur établissement d'affectation. De même, on a procédé à la pérennisation des « enseignants-paysans » dans leur village.

Bien que ces mesures aient obtenu des résultats encourageants, elles se heurtent néanmoins à des rejets de la part des enseignants comme des étudiants. En fait, on considère généralement que la profession enseignante demande beaucoup d'énergie pour un salaire et un statut social qui restent insuffisants, en particulier au niveau du primaire. Non seulement beaucoup de jeunes ne veulent pas entrer dans les métiers de l'enseignement, mais bien des enseignants déjà en fonction cherchent à quitter la profession. Ainsi l'écart entre les besoins qui sont immenses et croissent sans cesse et l'évolution des mentalités liée à la nouvelle donne économique ne permettra pas de résoudre ces problèmes de si tôt.

On aura compris que la problématique de l'éducation en Chine se situe certes dans une longue tradition culturelle. Pourtant, au cours des dernières décennies, et en particulier après 1949, l'éducation a vécu entre développement, stagnation et parfois régression (période de la "révolution culturelle»). Les actuelles conditions de développement économique et social, l'attention portée par le gouvernement à l'éducation, devraient favoriser une certaine progression. Cependant le passage à «l'économie socialiste de marché » a incité les mentalités chinoises à minorer les problèmes d'éducation au profit des tendances consuméristes et monétaristes. Il ne sera pas facile d'atteindre en l'an 2010 l'objectif fixé par l'État. 


\section{NOTES}

1. La Chine compte vingt-trois provinces en incluant celle de Chonqing créée en mars 1997, cinq régions autonomes et trois municipalités : Beijing, Shanghai, Tianjin.

2. Voir ci-dessous, « Dans l'enseignement supérieur ».

3. Nombre d'étudiants en France : 1463371 (1995).

\section{RÉSUMÉS}

La Chine est confrontée aux difficultés d'évolution d'un système éducatif lourd. Les objectifs principaux en sont : développer la décentralisation et l'autonomie tout en limitant les disparités régionales ; améliorer le recrutement, le statut et la formation des enseignants ; moderniser un enseignement supérieur, inspiré du modèle soviétique et relativement obsolète aujourd'hui; enfin, faire évoluer l'enseignement général dominé par le poids des examens.

\section{INDEX}

Mots-clés : décentralisation, enseignant, enseignement supérieur, politique éducative, réforme de l'enseignement, système éducatif

Index géographique : Chine

\section{AUTEURS}

\section{KECHAO XING}

Professeur à l'université Normale et à l'Institut des langues étrangères, Beijing, Chine. Professeur à l'université de Beijing et à l'Institut des langues étrangères. Auteur de L'éducation en France après la Deuxième Guerre mondiale (en chinois), Beijing, 1993.

\section{PIERRE-LOUIS GAUTHIER}

Inspecteur d'académie honoraire, France 